\title{
Ils vont bien... un an après
}

Marina Cavazzana-Calvo, Salima Hacein-Bey-Abina, Françoise Le Deist, Alain Fischer

$>$ Depuis la première publication [1] concernant l'essai clinique de thérapie génique réalisé à l'hôpital Necker (Paris, France), un certain nombre de questions restaient sans réponse. Elles concernaient différents champs tels que l'immunologie, l'hématopoìèse, la clinique et la place de ce type d'essai dans la stratégie thérapeutique du déficit immunitaire combiné sévère $(\mathrm{SCID}-\mathrm{XI})$ lié à I'X $(\rightarrow)$. Nous avons tenté d'y répondre par

$(\rightarrow) \mathrm{m} / \mathrm{s}$ 2000, n'5, p. 681 l'analyse détaillée des caractéristiques des cinq premiers patients traités avec un recul de 1 à 2 ans et demi [2] et des patients traités par la suite.

\section{Les résultats cliniques}

\section{concernant initialement deux patients}

\section{sont-il reproductibles?}

Neuf patients atteints de SCID-XI ont été traités depuis mars 1999 : huit d'entre eux présentaient une forme typique, associant un tableau clinique évocateur et une absence de lymphocytes $T$ et natural killer (NK), un patient adolescent présentait une forme atypique. Chez sept des huit patients atteints d'un SCID-X1, le traitement par thérapie génique a restauré un nombre correct de lymphocytes $T$ dont la fonction est normale. Ils mènent une vie normale au sein de leur famille, l'un d'entre eux fréquente l'école maternelle. Les résultats cliniques et biologiques obtenus chez ces patients démontrent la reproductibilité de cette méthode thérapeutique tant en termes d'efficacité de transduction ex vivo des cellules $\operatorname{CD}^{+} 4^{+}$(20 à $40 \%$ ), que de cinétique d'apparition des lymphocytes T. En effet, si des cellules mononu- cléées exprimant le transgène peuvent être détectées chez les patients dès le $15^{\mathrm{e}}$ jour après l'injection des cellules génétiquement modifiées, à partir de cette date, le nombre de lymphocytes $T$ augmente progressivement et atteint des valeurs normales pour l'âge entre le troisième et le quatrième mois après la thérapie génique. La distribution des différentes sous-populations lymphocytaires $T \alpha \beta$ ou $\gamma \delta$ et $\operatorname{CD}^{+}$ou $\mathrm{CD}^{+}$est normale. Cette cinétique de reconstitution immunitaire est identique chez tous les patients et n'est pas modifiée par une infection virale grave comme la varicelle, ou une lymphoprolifération liée à un virus du groupe herpès observée chez tous les patients. Les caractéristiques fonctionnelles de ces lymphocytes $T$ sont comparables à celles des sujets normaux y compris quand on les évalue par des tests quantitatifs, que ce soit la mesure de la fréquence des lymphocytes $T$ prolifératifs (HPLT), ou des lymphocytes capables de produire I'IFN- $\gamma$ (interféron) ou I'IL-4.

Cependant, nous avons observé un échec : il s'agit d'un enfant âgé de 7 mois dont le diagnostic a été posé lors d'une BCGite disséminée avec adénopathies diffuses et hépatosplénomégalie déclenchée par la vaccination par le BCG. Une pneumopathie à Pneumocystis carinii et à entérovirus aggravait le tableau clinique. Au décours de la thérapie génique, une amélioration clinique a pu être observée mais seuls des lymphocytes NK transduits ont été détectés dans la circulation sanguine. L'hypersplénisme a conduit à la splénectomie 4 mois après le traitement; l'étude de la
Inserm U.429,

Hôpital Necker,

Pavillon Kirmisson,

149 , rue de Sèvres,

75015 Paris, France.

rate a montré la présence de cellules NK et de lymphocytes $B$ exprimant le transgène. Il est probable que l'hypersplénisme a été responsable de la rétention des cellules hématopoïétiques transduites. Le risque d'un échec de la prise de greffe lié à la présence d'une rate volumineuse au moment d'une greffe de moelle osseuse allogénique est connu, mais l'évaluation du risque opératoire lorsque la situation clinique est précaire et des conséquences à long terme de la splénectomie compliquent souvent l'indication de cet acte. Après splénectomie, cet enfant a reçu une greffe de moelle osseuse allogénique à partir d'un donneur non apparenté, greffe qui a permis de restaurer, au moins partiellement, son système immunitaire.

\section{Quelle est la durée de la correction} phénotypique observée?

Cette question est complexe car plusieurs mécanismes différents pourraient rendre compte d'un déclin de l'effet bénéfique observé au cours du temps. Parmi ceux-ci, la répression immédiate ou secondaire de la transcription du transgène qui s'est intégré au hasard dans le génome [3], une fonction thymique altérée chez ces patients, ou encore une absence de transduction des cellules hématopoïétiques les plus primitives, c'est-à-dire ayant le plus fort potentiel d'autorenouvellement. Les résultats pré-cliniques précédemment rapportés montraient que l'expression du transgène $\gamma_{c}$ n'était réprimée ni dans les lymphocytes $B$ des patients transformés par l'EBV et cultivés pendant $l$ an in vitro [4], ni dans les lymphocytes des souris 
$\gamma_{c^{-/-}}$traitées par transfert génique [5]. $\varepsilon n$ accord avec ces données pré-cliniques, aucun phénomène de répression du transgène n'a pu être mis en évidence dans les différentes cellules matures circulantes étudiées chez les patients traités. Il est possible que l'expression de $\gamma_{c}$ exerce une pression de sélection qui permette la survie et/ou le développement des cellules exprimant cette sous-unité membranaire. Un rôle éventuel du vecteur rétroviral utilisé ne peut pas être écarté non plus [3].

La fonction thymique de ces patients a pu être étudiée grâce à la quantification des cellules ayant récemment quitté le thymus après s'y être différenciées, et que l'on appelle les «émigrants » thymiques précoces. Cela se fait par la recherche, dans la population de lymphocytes $T$, de cellules porteuses de cercles d'excision d'ADN épisomique (TREC, $T$ receptor excision circles) provenant de la recombinaison des segments $V(D) J$ du récepteur T de l'antigène. Trois ans après la thérapie génique, le nombre de TREC reste comparable à celui des sujets témoins du même âge ; cette donnée, associée à la persistance d'un thymus de taille normale et d'un nombre normal de lymphocytes naïs, sont autant d'éléments en faveur d'une fonction thymique normale et persistante dans le temps chez ces patients. L'absence d'une réponse allogénique dont l'épithélium thymique serait la cible pourrait expliquer la persistance de la fonction thymique observée, contrairement à ce qui a été rapporté après allogreffe de cellules souches hématopoïétiques [6].

Si seul un nombre limité de précurseurs engagés vers les lignées lymphoïdes a pu être transduit ex vivo par cette technique, une diminution du nombre de lymphocytes $T$ au cours du temps pourrait survenir. Un certain optimisme peut néanmoins être de mise car, parallèlement à l'observation du maintien de la fonction thymique, nous avons accumulé un certain nombre de données qui suggèrent que ce sont bien des progéni- teurs hématopoiétiques très proches de ce que pourrait être une cellule « souche » qui ont été transduits.

$\varepsilon n$ effet, chez deux patients, 13 et 21 mois après le traitement, environ $1 \%$

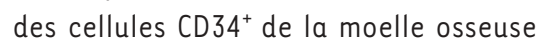
et entre 1 et $5 \%$ des progéniteurs de type LTC-IC (long term culture - initiating cells) [1] expriment le transgène. Cela est en accord avec l'observation d'une expression faible, mais stable dans le temps, de $\gamma$ c par les polynucléaires et les monocytes circulants. Ces résultats indiquent que des progéniteurs multipotents ont été transduits et sont détectables 2 ans après le traitement. Leur fréquence relativement élevée suggère que l'expression de $\gamma_{c}$ permet leur expansion.

\section{SCID-X1 : la place de la thérapie génique par rapport à la greffe de moelle osseuse allogénique} Malgré ses excellents résultats, la thérapie génique reste l'approche thérapeutique de deuxième choix après la greffe intra-familiale HLA géno-identique en raison de la cinétique de la reconstitution immunitaire liée à l'expansion des lymphocytes $T$ matures $d u$ donneur et des certitudes acquises quant aux résultats globaux au long cours de ce type de greffe.

Il y a, en revanche, de bonnes raisons de proposer de substituer la thérapie génique à la greffe allogénique lorsque la compatibilité HLA n'est que partielle. Le fait que la reconstitution lymphocytaire $T$ soit plus rapide après thérapie génique, d'environ deux mois, et que la correction du déficit de production des immunoglobulines soit, semble t-il, plus fréquente, plaident en faveur de la thérapie génique. Compte tenu de cette dernière donnée, on peut envisager d'utiliser la thérapie génique pour corriger le déficit de production d'immunoglobulines observé chez la majorité des enfants traités pour SCID-XI par greffe de moelle osseuse HLA haplo-incompatible. Une étude américaine, abordant cette question, est prévue à court terme. Compte tenu de la compétition potentielle avec la lymphopoïèse $B$ endogène présente (et même augmentée) chez ces patients, un traitement suppresseur des lymphocytes B et éventuellement des précurseurs pourrait s'avérer nécessaire.

Après 3 ans de recul, le bilan de cet essai de thérapie génique est encourageant. La persistance de l'efficacité thérapeutique, et l'absence d'effets toxiques observés, incitent à étendre son principe, fondé sur l'avantage sélectif conféré aux cellules transduites, au traitement d'autres formes de SCID comme les déficits en protéines Rag-1, Rag-2 ou Artemis. $\nabla$ One year after, they are ok

\section{RÉFÉRENCES}

1. Cavazzana-Calvo $M$, Hacein-Bey S, de Saint Basile G, et al. Gene therapy of human severe combined

immunodeficiency (SCID)$\mathrm{X} 1$ disease. Science 2000 ; $288: 669-72$.

2. Hacein-Bey-Abina $S$, Le Deist F, Carlier F, et al. Sustained correction of $X$ linked severe combined immunodeficiency by ex vivo gene therapy. $N$ EnglJ Med 2002 ; 346 : 1185-93.
3. Baum C, Richters A, Ostertag W. Retroviral vector-mediated gene expression in hematopoietic cells. Curr Opin Mol Ther 1999 ; 1 : 605-12.

4. Hacein-Bey H, CavazzanaCalvo M, Le Deist F, et al. Gamma-c gene transfer into SCID XI patients' Bcell lines restores normal high-affinity interleukin-2 receptor expression and function. Blood 1996; 87 : 3108-16.
5. Soudais C, Shiho T, Sharara LI, et al. Stable and functional lymphoid reconstitution of common cytokine receptor gamma chain deficient mice by retroviral-mediated gene transfer. Blood 2000 ; 95 3071-7.

6. Buckley RH, Schiff SE, Schiff RI, et al. Hematopoietic stem-cell transplantation for the treatment of severe combined immunodeficiency. $N$ Engl J Med 1999 ; 340 : 508-16. 